\title{
Neuropathic Corneal Pain Following LASIK Surgery: A Retrospective Case Series
}

\author{
Majid Moshirfar (D) - Udit M. Bhavsar · Kathryn M. Durnford • \\ Shannon E. McCabe · Yasmyne C. Ronquillo · Adam L. Lewis · \\ Phillip C. Hoopes
}

Received: May 4, 2021 / Accepted: May 28, 2021 / Published online: June 15, 2021

(c) The Author(s) 2021

\section{ABSTRACT}

Introduction: Transient dry eye symptoms have been reported following laser in situ keratomileusis (LASIK). Very rarely, patients may present with debilitating symptoms of dry eye syndrome (DES) with limited or no evidence of ocular surface disease. These patients are diagnosed with a form of DES known as neuropathic corneal pain (NCP).

Patients and Methods: This study is a retrospective medical record review of a case series of 18 patients who developed NCP post-LASIK over the years 1996-2021. All patients who

M. Moshirfar ( $₫)$. S. E. McCabe · Y. C. Ronquillo · P. C. Hoopes

HDR Research Center, Hoopes Vision, University of Utah Health Sciences Center, 11820 S. State Street

Suite \#200, Draper, UT 84020, USA

e-mail: cornea2020@me.com

M. Moshirfar

Department of Ophthalmology and Visual Sciences, John A. Moran Eye Center, University of Utah

School of Medicine, Salt Lake City, UT, USA

U. M. Bhavsar · K. M. Durnford

University of Utah School of Medicine, Salt Lake City, UT, USA

S. E. McCabe

Mission Hills Eye Center, Pleasant Hill, CA, USA

A. L. Lewis

Kansas City University of Medicine and Biosciences, Kansas City, MO, USA developed severe ocular pain following LASIK consistent with NCP were included. Patients with severe ocular pain who had evidence of severe ocular surface disease or other ophthalmic etiology to explain their debilitating symptoms were not included.

Results: The average age of patients in our study was 39.5 years. The majority of our patients were female (72.2\%) and of Caucasian ancestry $(83.3 \%)$. The average onset of symptoms was 9.6 months post-LASIK. Patients had past medical histories significant for neuropsychiatric conditions (50\%), functional pain syndromes (22.2\%), autoimmune diseases (33.3\%), and hypothyroidism (27.8\%), and the occurrence of these was higher than the national prevalence of these diseases. Symptoms were consistent with the severity and characteristics defining NCP. Treatment was multimodal, involved topical and systemic therapies, and was unique to each patient. Overall, the majority of patients had clinical improvement in symptoms following treatment with regular follow-up.

Conclusion: Although rare, the 26-year prevalence of NCP post-LASIK in our study was roughly 1 in 900 cases. The mean time to onset after surgery was delayed at 9.6 months. Certain risk factors such as neuropsychiatric conditions, history of functional pain syndromes, history of autoimmune conditions, and hypothyroidism may predispose patients to the development of this condition. Patients benefited from proper 
diagnosis and a multimodal approach to treatment.

Keywords: Cornea; Corneal neuralgia; Dry eye; Eye pain; Laser in situ keratomileusis; LASIK; Ocular neuropathic pain syndrome; Refractive surgery

\section{Key Summary Points}

Why carry out this study?

The prevalence of neuropathic corneal pain (NCP) following LASIK has not been documented, and our study identifies 18 patients out of roughly 16,000 LASIK patients seen over 26 years. The study discusses demographics, past medical history, symptoms and signs, clinical presentation, and treatments for these 18 individuals to contrast the current knowledge of this disease following LASIK.

\section{What was learned from this study?}

The prevalence of NCP post-LASIK from the years 1996 to 2021 was estimated at 1 in 900 patients. Our population emphasized that patients often present with a variety of symptoms and risk factors and require a unique, multimodal therapeutic approach to improve symptoms.

\section{DIGITAL FEATURES}

This article is published with digital features, including a summary slide, to facilitate understanding of the article. To view digital features for this article go to https://doi.org/10.6084/ m9.figshare.14697312.

\section{INTRODUCTION}

Laser in situ keratomileusis (LASIK) is one of the most common procedures performed worldwide, and around one million Americans will undergo LASIK each year [1-3]. Studies have shown that roughly $95 \%$ of patients will report dry eye symptoms characteristic of dry eye syndrome (DES) immediately after treatment [4-6], and this number diminishes to $28 \%$ at around 3 months [7]. DES post-LASIK is better described as a spectrum of disease that includes tear dysfunction, neurotrophic keratopathy, and dysesthetic cornea [8]. A subset of patients with DES post-LASIK have reported persistent, disabling symptoms of pain, spontaneous burning, light sensitivity, sensitivity to air, soreness, and achiness in the setting of either limited or lack of clinical signs of dry eye, and have limited response to conventional dry eye therapies alone $[9,10]$. The presentation of this subset of patients has come to be known as corneal neuralgia, ocular neuropathic pain, or neuropathic corneal pain (NCP) [11]. This is an extremely rare condition, and previous studies have only reported cases of NCP post-LASIK and have not been able to identify the incidence of this condition after refractive surgery. The aim of this study is to present a 26-year case series in an ophthalmologist's clinic where LASIK was performed on 600-700 patients annually. We discuss the demographics, relevant past medical history, onset of symptoms following LASIK, symptoms at presentation, clinical exam findings, and treatments tried within this population.

\section{METHODS}

This study followed the tenets of the Helsinki Declaration of 1964 and its later amendments. The study was Health Insurance Portability and Accountability Act of 1996 (HIPAA)-compliant and was approved by the institutional review board (IRB) of the Biomedical Research Alliance of New York (BRANY, Lake Success, NY, USA) in accordance with research standards and state law. This study is a retrospective case series of 18 patients with NCP following LASIK that was 
performed by a single surgeon at two tertiary eye care centers on 16,000 patients during the years 1996-2021. The LASIK flap was created with either a femtosecond laser or mechanical microkeratome, and there were no postoperative complications for any of the patients identified in the case series. All of the patients were from the Intermountain West region. Any patient who presented with symptoms of NCP following LASIK and a slit-lamp examination (SLE) of the cornea that was either normal, lacking in any corneal pathology, or was limited to mild ocular surface disease (mild to moderate meibomian gland dysfunction, tear breakup time less than $10 \mathrm{~s}$ and a Schirmer's test of less than $10 \mathrm{~mm}$ ) was included in this case series. The key unifying symptom was severe ocular pain, present in all patients. This symptom was measured subjectively and was assessed from chart records in which patients had described their surface eye pain with modifiers such as "extremely sensitive," "severe," or "constant." In addition to the treatments prescribed following diagnosis of NCP, all patients were treated with a topical third- or fourth-generation fluoroquinolone four times per day and a topical steroid four times a day for 1 week following LASIK. Data extracted from the medical records included patient demographics, past medical history, onset of symptoms post-LASIK, symptoms at presentation, clinical evaluation, and treatments utilized.

\section{RESULTS}

A summary of the patient demographics can be found in Table 1 . The patients' symptoms and clinical presentation are documented in Tables 2 and 3, respectively. Table 4 discusses the different treatment modalities employed for each of the 18 patients. Three out of the 18 cases are discussed in more detail.

\section{Case 1}

A 28-year-old African American man with a past medical history of meibomian gland dysfunction (MGD), DES, soft contact lens use, anxiety, depression, attention-deficit/hyperactivity
Table 1 Demographics and backgrounds of patients with neuropathic corneal pain post-LASIK

Demographics

Mean age (range)

39.5 years

(22-62 years)

SD 11.4

Gender

$\begin{array}{ll}\text { Female }(n=13) & 72.2 \% \\ \text { Male }(n=5) & 27.8 \%\end{array}$

Ethnicity

Caucasian $(n=15) \quad 83.3 \%$

Hispanic $(n=1) \quad 5.6 \%$

African American $(n=1) \quad 5.6 \%$

Asian $(n=1) \quad 5.6 \%$

Past medical history

Neuropsychiatric condition $(n=9) \quad 50.0 \%$

Depression $(n=7) \quad 38.9 \%$

Anxiety $(n=2) \quad 11.1 \%$

Bipolar disorder $(n=1) \quad 5.6 \%$

Panic disorder $(n=1) \quad 5.6 \%$

$\operatorname{ADHD}(n=1) \quad 5.6 \%$

Functional pain syndromes $(n=4) \quad 22.2 \%$

Fibromyalgia $(n=2) \quad 11.1 \%$

Chronic fatigue syndrome $(n=1) \quad 5.6 \%$

Chronic back pain $(n=1) \quad 5.6 \%$

Autoimmune conditions $(n=6) \quad 33.3 \%$

Hypothyroid disease $(n=5) \quad 38.5 \%$

LASIK flap type

Femtosecond laser $(n=15) \quad 83.3 \%$

Mechanical microkeratome $(n=3) \quad 16.7 \%$

Onset of symptoms

Median onset 6 months

Mean onset (range) $\quad 9.6$ months

(2-24 months) 
Table 1 continued

\section{SD 7.3}

$n=18$ adults; $A D H D$ attention-deficit/hyperactivity disorder

disorder (ADHD), and panic disorder underwent LASIK surgery with a femtosecond laser flap created with a superior hinge, thickness of 100 microns and diameter of $8.8 \mathrm{~mm}$ for the correction of $-6.50 \mathrm{D}$ with $+1.00 \mathrm{D}$ of oblique astigmatism in both eyes. Following the procedure, the patient's uncorrected distance visual acuity (UDVA) was 20/20 in both eyes. The procedure was uncomplicated, and the postoperative course was unremarkable. The patient presented to the clinic 6 months after the LASIK procedure with excruciating ocular pain, burning sensation, and photophobia. Clinical evaluation revealed normal SLE with no flap or tomography issues, lack of fluorescein staining, and presence of MGD. He was treated with a soft bandage contact lens, preservative-free dexamethasone eye drops, cyclosporine eye drops, preservative-free artificial tears, and punctal plugs. He was under treatment for his neuropsychiatric conditions with sertraline and amphetamine salts prior to LASIK. The patient felt the most benefit from bandage contact lenses and had significant improvement in his

Table 2 Symptoms and signs for patients with neuropathic corneal pain post-LASIK

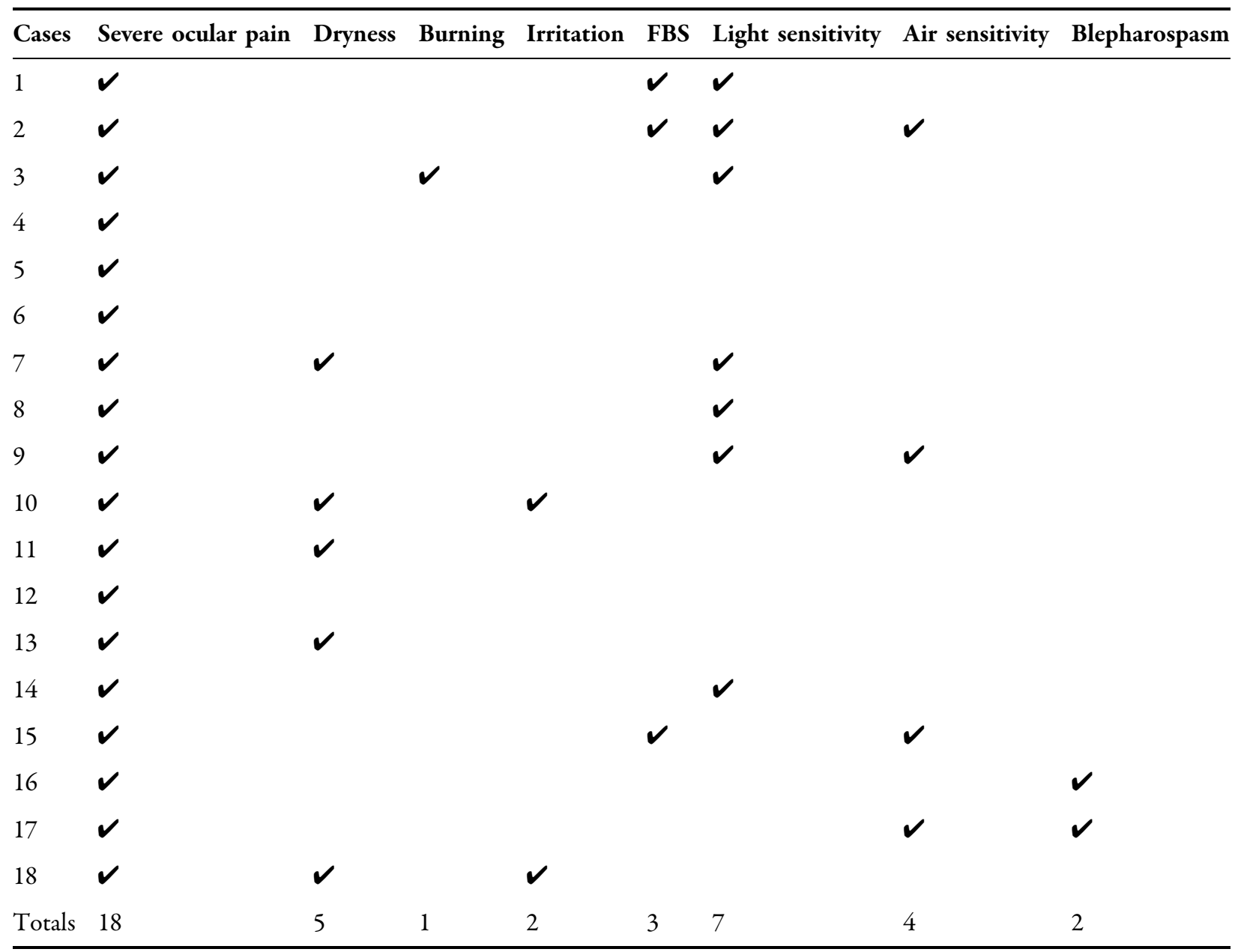

FBS foreign body sensation 
Table 3 Clinical presentation and characteristics of patients with neuropathic corneal pain post-LASIK

\begin{tabular}{|c|c|c|c|c|c|c|}
\hline Cases & $\begin{array}{l}\text { Corneal } \\
\text { staining }\end{array}$ & $\begin{array}{l}\text { Conjunctival } \\
\text { staining }\end{array}$ & MGD & $\begin{array}{l}\text { Tear breakup time } \\
(\text { in } s)^{a}\end{array}$ & $\begin{array}{l}\text { Schirmer's test I (in } \\
\mathrm{mm})^{\mathbf{b}}\end{array}$ & $\begin{array}{l}\text { Tetracaine } \\
\text { challenge test }{ }^{c}\end{array}$ \\
\hline 1 & 0 & 0 & Mod & & & \\
\hline 2 & 0 & 0 & Mild & $>10$ & $>10$ & Neg \\
\hline 3 & 0 & 0 & None & $<10$ & $>10$ & \\
\hline 4 & Trace & 0 & Mild & & & \\
\hline 5 & Trace & $2+$ & Mod & $<10$ & $>10$ & Pos \\
\hline 6 & 0 & 0 & None & & & \\
\hline 7 & 0 & 0 & Mild & & & \\
\hline 8 & 0 & 0 & Mild & & & \\
\hline 9 & Trace & 0 & Mild & & & \\
\hline 10 & 0 & 0 & None & & & \\
\hline 11 & Trace & Trace & Mod & $>10$ & $>10$ & Pos \\
\hline 12 & 0 & $1+$ & Mod & $>10$ & $>10$ & Pos \\
\hline 13 & $1+$ & $1+$ & Mild & $>10$ & $>10$ & \\
\hline 14 & Trace & Trace & Mod & & & $\mathrm{Neg}$ \\
\hline 15 & 0 & 0 & Mild & $<10$ & $>10$ & $\mathrm{Neg}$ \\
\hline 16 & 0 & 0 & Mild & & & Neg \\
\hline 17 & 0 & 0 & Mild & & & Neg \\
\hline 18 & 0 & 0 & Mild & & & \\
\hline
\end{tabular}

MGD meibomian gland dysfunction, $M o d$ moderate, Neg negative, Pos positive. Blank cells indicate that the test was not performed and/or not documented

Staining was graded on a standard grading system where 0 is no staining and $4+$ is severe staining

a,b Tear breakup time (TBUT) and Schirmer's test I are diagnostic tools for dry eye. TBUT greater than $10 \mathrm{~s}$ is considered normal, and greater than $10 \mathrm{~mm}$ is considered normal for the Schirmer's test I

c The tetracaine challenge assesses the peripheral versus central sensitization of pain, and a positive result is when there is a reduction in pain after application of tetracaine drops

symptoms from the multimodal treatment. Following improvement in his symptoms, he completed a bachelor's degree in computer sciences from an Ivy League school.

\section{Case 3}

A 22-year-old Caucasian man with a past medical history of depression with suicidal ideation underwent LASIK surgery. The LASIK flap was created with a mechanical microkeratome with a superior hinge and flap thickness of 160 microns for the correction of $-2.00 \mathrm{D}$ in the right eye (OD) and $-1.75 \mathrm{D}$ in the left eye (OS). The procedure was uncomplicated, and the immediate postoperative course was unremarkable. His post-procedure UDVA in both eyes was $20 / 15$. The patient presented to the clinic 6 months after LASIK with symptoms of ocular pain, photophobia, and foreign body sensation in both eyes. Clinical examination revealed 
682

Ophthalmol Ther (2021) 10:677-689

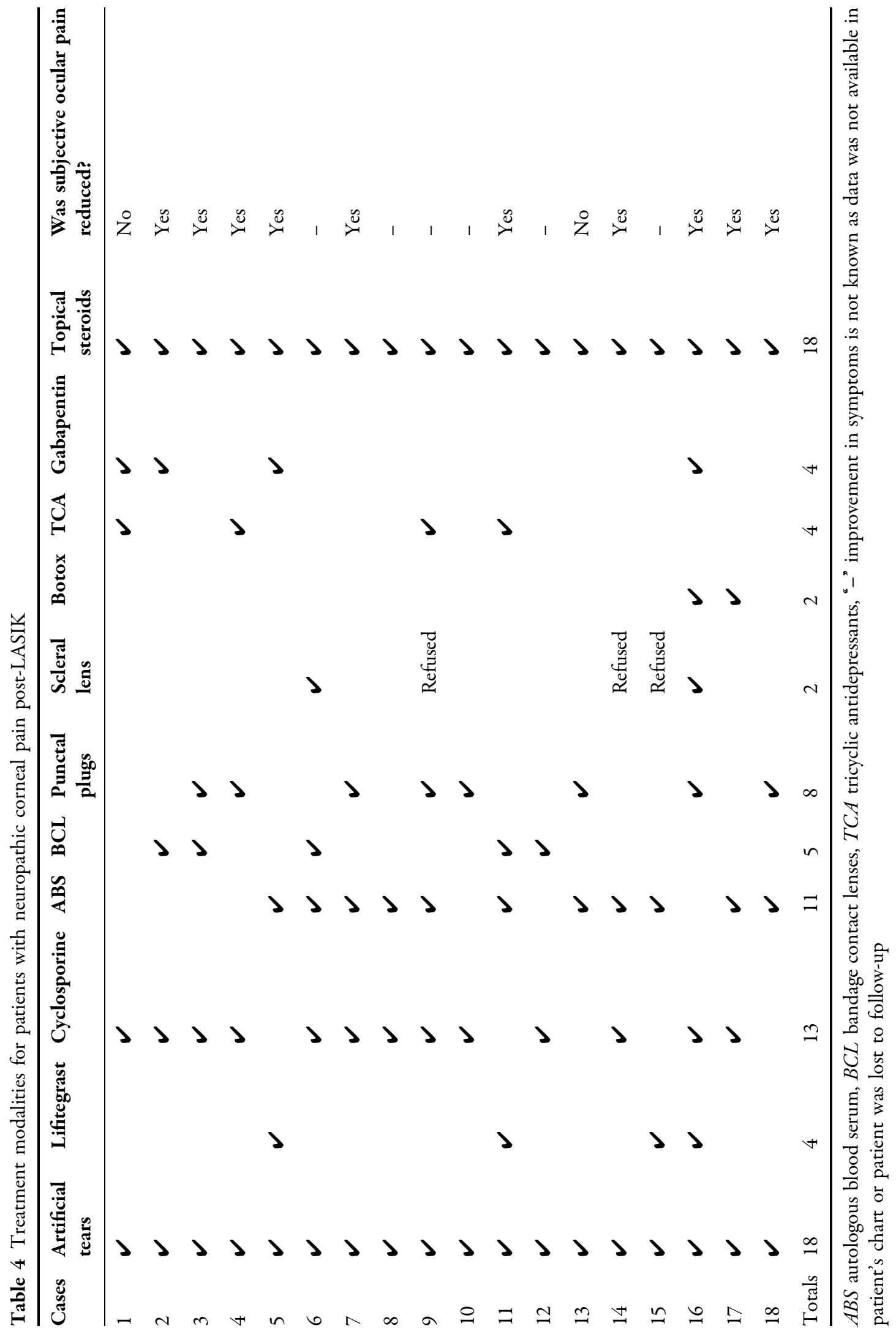

$\triangle$ Adis 
normal SLE, lack of fluorescein staining, and normal corneal tomography and topography. There was no evidence of MGD. The patient was treated with cyclosporine, preservative-free artificial tears, topical steroids, gabapentin, and a tricyclic antidepressant (TCA). The patient was first diagnosed with depression in high school, and he had a history of suicidal ideation prior to surgery. He was also under treatment for his depression with fluoxetine and other psychotropic medications before having LASIK. Despite the initiation of various treatments, he did not notice any improvement in his ocular symptoms after treatment initiation and committed suicide prior to the 1-year follow-up post-LASIK.

\section{Case 18}

A 36-year-old Caucasian woman with a past medical history of asthma underwent an uncomplicated LASIK procedure with a femtosecond laser flap created with a superior hinge, thickness of 115 microns, and a diameter of $8.3 \mathrm{~mm}$ and correction of $-5.50 \mathrm{D}$ OD and $-5.75 \mathrm{D}$ OS. Her postoperative course was unremarkable, and post-procedure UDVA in both eyes was 20/20. She reported to the clinic 9 months after her LASIK procedure with symptoms of stabbing ocular pain, sensation of dryness, irritation, blurry vision, sensitivity to moving air, and severe headaches. She noted difficulty sleeping and performing daily tasks as a result of her ocular pain. Clinical examination at this point revealed unchanged visual acuity, normal SLE, normal corneal staining, and mild MGD. In vivo confocal microscopy (IVCM) of the cornea was obtained on initial presentation and showed decreased nerve density, increased nerve beading, and presence of microneuromas (MNs). Following the diagnosis of NCP, she was treated using loteprednol eye drops, fluorometholone eye drops, preservative-free artificial tears, autologous blood serum (ABS), and punctal plugs. Treatment with nortriptyline, a TCA, was also recommended, but the patient refused. She was followed in the clinic regularly and started noticing improvement in her symptoms 5 months after initiation of treatment.

\section{Demographics}

Out of the approximately 16,000 cases of LASIK performed by a single surgeon from January 1996 to April 2021, we were able to identify 18 instances of NCP that occurred post-procedure. The occurrence of NCP is $0.0011 \%$ or approximately 1 in every 900 LASIK cases from our sample. All of these patients underwent an uncomplicated LASIK procedure. The mean age of the patients was 39.5 years (range 22-62 years), with a female-to-male ratio of 13:5. Of the 18 cases, $15(83.3 \%)$ individuals identified as Caucasian, one identified as African-American (5.6\%), one identified as Hispanic (5.6\%), and one as Asian (5.6\%). The mean time to onset of symptoms was 9.6 months (range 2-24 months) post-LASIK. The median time to onset of symptoms post-LASIK was lower, at 6.0 months. (Table 1).

\section{Past Medical History}

All patients had uncomplicated LASIK surgeries with optimal visual outcomes; three underwent mechanical microkeratome LASIK (16.7\%) and the rest had LASIK performed via femtosecond laser $(83.3 \%)$. Prior to undergoing LASIK, three $(16.7 \%)$ individuals had been diagnosed with DES, nine $(50.0 \%)$ patients had a history of neuropsychiatric disorders, four (22.2\%) patients had been diagnosed with functional pain syndrome, six (33.3\%) patients had a history of an autoimmune condition, and five $(27.8 \%)$ had a history of hypothyroidism. Of the nine individuals with a neuropsychiatric history, one had anxiety; six had only depression diagnosed; one patient had depression, anxiety, ADHD, and panic disorder; and one patient had a diagnosis of depression and bipolar disorder. Out of the four individuals diagnosed with functional pain syndrome, two had fibromyalgia, one had chronic fatigue syndrome, and one had chronic back pain. Of the six individuals with a history of autoimmune conditions, two had Hashimoto's, one had diabetes and psoriasis, two had arthritis, and one had alopecia. It is important to note that all 
patients who presented with comorbid systemic or neuropsychiatric conditions were being actively treated by another physician for these conditions. For example, all patients with a history of depression were actively managed by another physician for that condition (Table 1).

\section{Clinical Presentation}

On initial presentation, 14 patients $(77.8 \%)$ reported experiencing multiple symptoms. All patients complained of severe ocular pain, five $(27.8 \%)$ had a sensation of dryness, one $(5.6 \%)$ reported burning sensation, two (11.1\%) reported irritation, three $(16.7 \%)$ had a foreign body sensation in their eyes, seven (38.9\%) reported photophobia, four (22.2\%) reported sensitivity to air, and two (11.1\%) reported new-onset blepharospasm (Table 2).

All patients underwent fluorescein staining, conjunctival staining, and meibomian gland functional studies corneal staining. The lack of fluorescein staining was noted in 12 (66.7\%) individuals, trace fluorescein staining was noted in five $(27.8 \%)$ individuals, and $1+$ fluorescein staining was noted in one $(5.6 \%)$ individual. Conjunctival staining showed that $13(72.2 \%)$ individuals had a lack of staining, two (11.1\%) individuals had trace staining, two $(11.1 \%)$ had $1+$ staining, and one $(5.6 \%)$ had $2+$ staining. Meibomian gland studies showed that three (16.7\%) individuals had no MGD, ten (55.6\%) had mild MGD, and five (27.8\%) had moderate MGD. Tear breakup time was performed in seven $(38.9 \%)$ patients and found to be abnormal in three of them $(<10 \mathrm{~s})$. Schirmer's test I was performed in seven (38.9\%) patients and was found to be normal in all of them $(>10 \mathrm{~mm})$. A tetracaine challenge was conducted in eight $(44.4 \%)$ individuals, three of whom noted improvement in pain, while five did not. (Table 3) UDVA in both eyes postLASIK was 20/20 or better in five patients, 20/30 or better in two patients, and 20/50 in one patient. The best-corrected visual acuity was $20 / 20$ or better for both eyes in all patients.

\section{Treatment}

All patients used preservative-free artificial tears and topical steroids (loteprednol or preservative-free $0.1 \%$ dexamethasone), eight (44.4\%) used ABS 20\%, two (11.1\%) used ABS $33 \%$, one $(5.6 \%)$ used ABS 50\%, 13 (72.2\%) used cyclosporine, four (22.2\%) used gabapentin, four $(22.2 \%)$ used TCA, four (22.2\%) used lifitegrast, three (16.7\%) used naltrexone, two $(11.1 \%)$ were given botox for management of blepharospasm, eight (44.4\%) used punctal plugs, five $(27.8 \%)$ utilized bandage contact lenses, and two (11.1\%) used scleral lenses (three [16.7\%] individuals also refused this treatment option) (Table 4).

Not shown in Table 4: two (11.1\%) individuals were treated with albumin $5 \%$, one $(5.6 \%)$ tried trigeminal nerve ganglion ablation, one (5.6\%) was managed with intense pulsed light (IPL) for MGD, and four (22.2\%) were managed with an electronic heating device such as LipiFlow ${ }^{\circledR}$ for MGD. Additionally, three of the nine individuals with a neuropsychiatric history were also treated for their condition with medications such as sertraline, fluoxetine, amphetamine salts, and trazodone. Finally, ten $(55.6 \%)$ patients reported that the therapy combination used to treat their ocular pain led to improvement in their symptoms (Table 4).

\section{DISCUSSION}

While it is an exceptionally uncommon condition, some patients may present post-LASIK with refractory dry eye symptoms characteristic of NCP. Our study identified 18 patients seen by a single surgeon over the course of 26 years presenting with persistent and severe symptoms of DES out of proportion to their clinical examination. Treatment for these patients consisted of a combination of dry eye therapies, anti-inflammatory medications, pain control, anticonvulsants, and management of comorbid neuropsychiatric conditions. Of the 18 , ten patients reported alleviation of their symptoms, two did not, and six were lost to follow-up. There is a paucity of data regarding the incidence of NCP post-LASIK treatment. From this case series, the prevalence of NCP post-LASIK is approximately 1 in every 900 cases, making it an extremely rare condition following LASIK surgery. 
Risk factors associated with the development of DES could also play a role in the development of NCP [8]. Multiple studies have shown that women are at an increased risk of developing DES post-LASIK [5, 12, 13], and this was consistent with what we observed in our case series. Asian ethnicity has also been associated with increased risk of developing DES after LASIK $[5,14]$. Interestingly, only one patient in our case series was of Asian ethnicity, and we observed that $83.3 \%$ of individuals who developed NCP post-LASIK identified as Caucasian. The population demographics of the region in which these individuals were diagnosed and treated are primarily Caucasian $(72.8 \%)$, with only $5.4 \%$ Asian [15]. This difference could explain why we observed that most individuals who developed NCP following LASIK were Caucasian.

Neuropsychiatric conditions such as depression, anxiety, and post-traumatic stress disorder (PTSD) have also been associated with an increased risk of developing DES [10, 16-19]. We noted that $50.0 \%$ of the patients in our case series had a history of neuropsychiatric conditions. Studies have also found worsening of neuropsychiatric conditions as a result of NCP $[2,20]$, and another study has also reported rare cases of suicide following laser refractive surgery [21]. Although worsening of symptoms of neuropsychiatric conditions was not measured in our patients, one individual (case 3) with a history of depression committed suicide within 1 year of onset of symptoms of NCP post-LASIK. Previous studies in patients with NCP postLASIK have not documented the prevalence of neuropsychiatric disease in their studies. However, a study of 181 patients diagnosed with neuropathic ocular pain showed that $43.6 \%$ were on an antidepressant and 40.9\% were taking an anxiolytic [2]. Another study of 59 patients with NCP documented that $16.7 \%$ of their patients had depression and $10 \%$ had anxiety [22]. The US Centers for Disease Control (CDC) reports that the percentage of adults above 18 years of age that report feelings of depression in the US population is $4.7 \%$ and the percentage that reports frequent feelings of anxiety is $11.2 \%$ [23]. Our study results, in conjunction with existing literature, further support the hypothesis that the presence of neuropsychiatric conditions can predispose individuals to the development of NCP. Understanding that underlying neuropsychiatric conditions may predispose the patient to NCP should prompt physicians who diagnose or suspect this condition to ask additional questions. The physician should inquire about a past medical history of neuropsychiatric disorders, confirm that these patients have a provider who is actively managing these conditions, and refer the patient to the appropriate psychologist or psychiatrist if they are indeed not being actively managed.

Fibromyalgia, other functional pain syndromes, autoimmune diseases, and thyroid diseases are some of the other systemic diseases shown to be linked to NCP [24]. Of the patients in our study, $22.2 \%$ had a diagnosis of functional pain syndrome, $33.3 \%$ had an autoimmune condition, and $27.8 \%$ had hypothyroidism. Other studies have shown the occurrence of autoimmune diseases in patients with NCP at $16.7 \%$, hypothyroid disease at $16.7 \%$, and fibromyalgia at $6.7 \%$ [22]. Of the patients with hypothyroid disease, two were known to have Hashimoto's thyroiditis. In the United States, fibromyalgia affects about $2 \%$ of the adult population [25], hypothyroidism is seen in $4.6 \%$ of the population 12 years and older [26], and autoimmune disease is present in $5-8 \%$ of the population [13]. There is no consensus in the literature regarding the prevalence of comorbidities in individuals with NCP, but the occurrence here is higher than the prevalence of the general US population.

The symptoms of NCP are nonspecific and can be similar to those seen with DES [13]. The literature reveals that those individuals with NCP report symptoms such as ocular pain, irritation, itchiness, dryness, grittiness, burning, aching, light sensitivity, soreness, and foreign body sensation $[9,13,24,27,28]$. Our case series is consistent with previous literature regarding symptoms associated with NCP. Ocular pain is the only consistent symptom we observed among our patients. Additionally, the literature shows that SLE of the cornea in individuals with NCP post-LASIK tends to be normal and will occasionally show dryness [13, 24, 27]. 
In our study, 15 (83.3\%) individuals had evidence of MGD and three (16.7\%) individuals had tear breakup time test results indicative of DES on clinical evaluation. Although most individuals in our study did have some evidence of minimal DES on clinical evaluation, the severity of the symptoms reported was out of proportion to what was seen on examination.

IVCM of the cornea may also be helpful in the diagnosis of NCP [9, 10, 13, 24, 27]. Studies have reported that this test tends to demonstrate the presence of MNs, increased nerve tortuosity and beading, decreased nerve fiber density, and a larger dendritic cell population in individuals with NCP $[9,24,27]$. IVCM of the cornea obtained on one of the patients (case 18) in our study at the time of diagnosis also showed decreased nerve density, increased nerve beading, and presence of MNs. However, it is worth noting that a recent study published demonstrated that only 16 out of 63 individuals with features of neuropathic pain had MNs on IVCM. Additionally, 10 out of 55 individuals with no features of neuropathic pain also had MNs on IVCM. Finally, the same study also found that the presence of MNs on IVCM among individuals with and without dry eye symptoms was not statistically significant [29]. This indicates that although the presence of MNs on IVCM may be helpful in the diagnosis of NCP, their absence does not rule out this condition.

Studies have previously shown that testing the changes in pain level after applying a topical anesthetic on the cornea can be used to differentiate between peripheral and central sensitization of pain in individuals with NCP $[10,13,24,27]$. The application of a topical anesthetic should reduce the transmission of peripheral pain. Therefore, patients who experience complete or partial resolution of pain suffer from peripheral pain or mixed peripheral and central pain, respectively. Individuals who do not notice any improvement in their symptoms suffer, at least in part, from central pain $[13,24]$. In our study, eight patients were evaluated with a topical tetracaine challenge test. Of these patients, five reported no improvement in their symptoms and three had complete but transient resolution of pain post-anesthetic application. This indicates that of those eight patients, five had a component of central sensitization of pain and three had either peripheral or mixed sensitization of pain. Utilizing this test to determine the etiology of NCP, central versus peripheral, can help guide treatment decisions $[13,24]$.

Variability in symptoms, along with a benign clinical examination, makes NCP a difficult condition to diagnose. It should be included in the differential diagnosis for any individual who reports severe ocular pain, burning, irritation, light and air sensitivity, and foreign body sensation with minimal or no evidence of ocular surface disease post-LASIK. Although symptoms of NCP overlap with those of DES, the severity reported by patients can point towards a diagnosis of NCP. In addition, the risk factors listed above may help raise clinical suspicion for the disease if other corneal diseases have been ruled out.

Literature suggests that the treatment approach of this condition should be multimodal [13]. Studies have reported successful management of NCP with artificial tears, ABS, anti-inflammatory agents (topical steroids, cyclosporine, anakinra, tacrolimus), antidepressants (TCAs, selective serotonin reuptake inhibitors [SSRIs], and serotonin-norepinephrine reuptake inhibitors [SNRIs]), anticonvulsants (gabapentin, carbamazepine, and pregabalin), pain medications (naltrexone or tramadol), contact lenses (scleral or soft bandage), vitamins (B12 and D), omega-3 fatty acids, exercise, and even electrical stimulation of the trigeminal ganglion [13, 24, 27, 30-35]. Each patient in our study had a unique multimodal treatment plan involving many of the treatments listed. Since all patients reviewed here were managed with more than one therapy, the relative contribution of each modality in improving the symptoms of ocular pain cannot be assessed. We believe that there is no single treatment approach, and most patients will require several modalities to achieve significant improvement of symptoms. Of the patients who were assessed, 83.3\% had improvement in their pain symptoms following multimodal treatment. Although these symptoms can be extraordinarily debilitating, 
especially when left untreated, most patients have significant improvement with regular follow-up. It is critical to recognize the validity of these patients' symptoms, despite there being limited or no evidence of corneal pathology related to dry eye, and begin treatment with regular follow-up. The majority of the patients presented with debilitating symptoms but were then able to return to normal activities of daily living following treatment.

While the data presented here today represents one physician's experience over a 26-year career performing LASIK, our study has some limitations. As with all retrospective analyses, there are limits in standardization. As the knowledge of best practices for treatment and diagnosis evolved, so did the diagnosis and treatment for the patients in this study. For this reason, and due to the rarity of the condition and the multimodal treatment approach for the disease, the treatment regimen could not be standardized amongst the patients. This lack of standardization included the lack of a pain scale such as the visual analog scale (VAS) or ocular pain assessment survey (OPAS). Because of the rarity of this disease, we suggest that future studies, especially any prospective studies, include a standardized pain scale in the assessment of ocular pain in the workup or diagnosis of NCP. The tetracaine challenge was performed on some patients for somatosensory assessment, but we realize that there are additional assessments of this nature available. Corneal esthesiometry measured with a device such as the Cochet-Bonnet contact esthesiometer was not done but would be suggested for future studies. As with other retrospective studies, we did not have controls. Additionally, there was incomplete data in some of the patients' charts and not all the data gathered was comparable between the cases. We attempted to estimate the prevalence of post-LASIK NCP over the 26 years, but this is based on the assumption that patients with NCP returned for follow-up outside the immediate postoperative period. The mean onset of symptoms in our patient population was 9.6 months, with the lower limit being 2 months; thus, the delay in onset could have led to increased loss of follow-up. Despite these limitations, the knowledge presented helps to elucidate general statistics regarding NCP in the post-LASIK population that have otherwise not been documented.

\section{CONCLUSION}

Dry eye symptoms are commonly reported after LASIK. NCP, however, is a rare presentation of debilitating, persistent dry eye symptoms with limited or no clinical evidence of dry eye. The pathophysiology of this condition remains unclear, but there appear to be risk factors related to demographics and past medical history that may predispose patients to this condition. The prevalence of NCP following LASIK over 26 years was roughly 1 in 900 . With early identification, regular follow-up, and multimodal treatment, most patients have significant improvement and go on to lead functional lives during which they manage the otherwise debilitating symptoms of NCP.

\section{ACKNOWLEDGEMENTS}

We thank the participants of the study.

Funding. This research did not receive any specific grant from funding agencies in the public, commercial, or not-for-profit sectors.

Authorship. All named authors meet the International Committee of Medical Journal Editors (ICMJE) criteria for authorship for this article, take responsibility for the integrity of the work as a whole, and have given their approval for this version to be published.

Authorship Contributions. Majid Moshirfar helped design the project and conducted the chart review. Adam L. Lewis, Udit M. Bhavsar, and Kathryn M. Durnford helped with the literature review. Udit M. Bhavsar and Kathryn M. Durnford designed the tables, organized the information gathered from patient charts, and wrote the manuscript. Finally, Majid Moshirfar, Yasmyne C. Ronquillo, Shannon E. McCabe, and Phillip C. Hoopes helped in the drafting and editing of the final manuscript. 
Disclosures. The authors Majid Moshirfar, MD; Udit M. Bhavsar; Kathryn M. Durnford; Shannon E. McCabe, MD; Yasmyne C. Ronquillo, MD; Adam L. Lewis; and Phillip C. Hoopes, MD have nothing to disclose.

Compliance with Ethics Guidelines. The study was Health Insurance Portability and Accountability Act of 1996 (HIPAA)-compliant and was an IRB-approved study by the Biomedical Research Alliance of New York (BRANY, Lake Success, NY) in accordance with research standards and state law.

Data Availability. Data sharing is not applicable to this article as no datasets were generated or analyzed during the current study.

Open Access. This article is licensed under a Creative Commons Attribution-NonCommercial 4.0 International License, which permits any non-commercial use, sharing, adaptation, distribution and reproduction in any medium or format, as long as you give appropriate credit to the original author(s) and the source, provide a link to the Creative Commons licence, and indicate if changes were made. The images or other third party material in this article are included in the article's Creative Commons licence, unless indicated otherwise in a credit line to the material. If material is not included in the article's Creative Commons licence and your intended use is not permitted by statutory regulation or exceeds the permitted use, you will need to obtain permission directly from the copyright holder. To view a copy of this licence, visit http://creativecommons.org/licenses/by$\mathrm{nc} / 4.0 /$.

\section{REFERENCES}

1. Solomon KD, de Castro FLE, Sandoval HP, Biber JM, Groat B, Neff $\mathrm{KD}$, et al. LASIK world literature review: quality of life and patient satisfaction. Ophthalmology. 2009;116(4):691-701.

2. Crane AM, Levitt RC, Felix ER, Sarantopoulos KD, McClellan AL, Galor A. Patients with more severe symptoms of neuropathic ocular pain report more frequent and severe chronic overlapping pain conditions and psychiatric disease. $\mathrm{Br} \mathrm{J}$ Ophthalmol. 2017;101(2):227-31.

3. Sutton G, Lawless M, Hodge C. Laser in situ keratomileusis in 2012: a review. Clin Exp Optom. 2014;97(1):18-29.

4. Chao C, Golebiowski B, Stapleton F. The role of corneal innervation in LASIK-induced neuropathic dry eye. Ocul Surf. 2014;12(1):32-45.

5. Shtein RM. Post-LASIK dry eye. Expert Rev Ophthalmol. 2011;6(5):575-82.

6. Yu EYW, Leung A, Rao S, Lam DSC. Effect of laser in situ keratomileusis on tear stability. Ophthalmology. 2000;107(12):2131-5.

7. Eydelman M, Hilmantel G, Tarver ME, Hofmeister EM, May J, Hammel K, et al. Symptoms and satisfaction of patients in the Patient-Reported Outcomes With Laser In Situ Keratomileusis (PROWL) studies. JAMA Ophthalmol. 2017;135(1):13-22.

8. Nettune GR, Pflugfelder SC. Post-LASIK tear dysfunction and dysesthesia. Ocul Surf. 2010;8(3): 135-45.

9. Theophanous C, Jacobs DS, Hamrah P. Corneal neuralgia after LASIK. Optometry Vis Sci. 2015;92(9):233-240.

10. Galor A, Moein H-R, Lee C, Rodriguez A, Felix ER, Sarantopoulos KD, et al. Neuropathic pain and dry eye. Ocul Surf. 2018;16(1):31-44.

11. Yuan J-H, Schulman BR, Effraim PR, Sulayman D-H, Jacobs DS, Waxman SG. Genomic analysis of 21 patients with corneal neuralgia after refractive surgery. Pain Rep. 2020;5(4):e826-e.

12. Shoja MR, Besharati MR. Dry eye after lasik for myopia: incidence and risk factors. Eur J Ophthalmol. 2007;17(1):1-6.

13. Moshirfar M, Benstead EE, Sorrentino PM, Tripathy K. Ocular neuropathic pain. Treasure Island: StatPearls Publishing; 2020.

14. Albietz JM, Lenton LM, McLennan SG. Dry eye after LASIK: Comparison of outcomes for Asian and Caucasian eyes. Clin Exp Optom. 2005;88(2):89-96.

15. QuickFacts Salt Lake City, Utah. United States Census Bureau Website. https://www.census.gov/ quickfacts/saltlakecitycityutah. Accessed 29 April 2021.

16. Galor A, Levitt RC, Felix ER, Martin ER, Sarantopoulos CD. Neuropathic ocular pain: an important yet underevaluated feature of dry eye. Eye (Lond). 2015;29(3):301-12. 
17. Galor A, Feuer W, Lee DJ, Florez H, Carter D, Pouyeh B, et al. Prevalence and risk factors of dry eye syndrome in a United States Veterans Affairs population. Am J Ophthalmol. 2011;152(3):377-84. e2.

18. Galor A, Feuer W, Lee DJ, Florez H, Faler AL, Zann $\mathrm{KL}$, et al. Depression, post-traumatic stress disorder, and dry eye syndrome: a study utilizing the National United States Veterans Affairs Administrative Database. Am J Ophthalmol. 2012;154(2): 340-6.e2.

19. Vehof J, Sillevis Smitt-Kamminga N, Nibourg SA, Hammond CJ. Predictors of discordance between symptoms and signs in dry eye disease. Ophthalmology. 2017;124(3):280-6.

20. Galor A, Covington D, Levitt AE, McManus KT, Seiden B, Felix ER, et al. Neuropathic ocular pain due to dry eye is associated with multiple comorbid chronic pain syndromes. J Pain. 2016;17(3):310-8.

21. Salimi A, Ing E, Nianiaris N. Suicide and laser refractive surgery. J Ophthal Vis Res (JOVR). 2020; 15(3):432-34.

22. Dieckmann G, Ozmen MC, Engert R, Hamrah P. Efficacy and Tolerability of Low-dose Naltrexone for Pain Relief among Neuropathic Corneal Pain Patients. Investig Ophthalmol Vis Sc. 2020;61(7): 4088.

23. Mental Health Center for Disease Prevention and Control Website [updated March 1, 2021]. https:// www.cdc.gov/nchs/fastats/mental-health.htm. Accessed 22 April 2021

24. Dieckmann G, Goyal S, Hamrah P. Neuropathic corneal pain: approaches for management. Ophthalmology. 2017;124(11S):S34-47.

25. Fibromyalgia Centers for Disease Control and Prevention Website [updated January 6, 2020]. https://www.cdc.gov/arthritis/basics/fibromyalgia. htm. Accessed 22 April 2021.

26. Hypothyroidism (Underactive Thyroid) National Institute of Diabetes and Digestive and Kidney
Diseases Website [updated August, 2016]. https:// www.niddk.nih.gov/health-information/ endocrine-diseases/hypothyroidism\#: :text= How\%20common\%20is\%20hypothyroidism\% $3 \mathrm{~F}$, although $\% 20$ most $\% 20$ cases $\% 20$ are $\% 20$ mild. \&text=That's\%20almost $\% 205 \% 20$ people $\% 20$ out $\%$ 20of\%20100. Accessed 22 April 2021.

27. Goyal S, Hamrah P. Understanding neuropathic corneal pain-gaps and current therapeutic approaches. Semin Ophthalmol. 2016;31(1-2):59-70.

28. Rosenthal P, Baran I, Jacobs DS. Corneal pain without stain: is it real? Ocul Surf. 2009;7(1):28-40.

29. Dermer H, Hwang J, Mittal R, Cohen AK, Galor A. Corneal sub-basal nerve plexus microneuromas in individuals with and without dry eye. $\mathrm{Br} \mathrm{J}$ Ophthalmol. 2021;0:1-7.

30. Aggarwal S, Colon C, Kheirkhah A, Hamrah P. Efficacy of autologous serum tears for treatment of neuropathic corneal pain. Ocul Surf. 2019;17(3): 532-9.

31. Sayegh RR, Sweet JA, Miller JP, Hayek SM. Electrical Stimulation of the Trigeminal Ganglion and Intrathecal Drug Delivery Systems for the Management of Corneal Neuropathic Pain. Cornea. 2016;35(4):576-77.

32. Shetty R, Deshpande K, Ghosh A, Sethu S. Management of Ocular Neuropathic Pain With Vitamin B12 Supplements: A Case Report. Cornea. 2015;34(10):1324-25.

33. Singman EL, Poon D, Jun AS. Putative corneal neuralgia responding to vitamin D supplementation. Case Rep Ophthalmol. 2013;4(3):105-8.

34. Mian SZ, Agranat JS, Jacobs DS. Prosthetic Replacement of the Ocular Surface Ecosystem (PROSE) Treatment for Complications After LASIK. Eye \& Contact Lens. 2016;42(6):371-73.

35. Chinnery HR, Naranjo Golborne C, Downie LE. Omega-3 supplementation is neuroprotective to corneal nerves in dry eye disease: a pilot study. Ophthalmic Physiol Opt. 2017;37(4):473-81. 